UC3M Working papers

Economics

$16-11$

July, 2016

ISSN 2340-5031
Departamento de Economía Universidad Carlos III de Madrid Calle Madrid, 126 28903 Getafe (Spain) Fax (34) 916249875

\title{
Equation-by-Equation Estimation of Multivariate Periodic Electricity Price Volatility*
}

\author{
Alvaro Escribano ${ }^{\dagger}$ and Genaro Sucarrat ${ }^{\ddagger}$
}

\begin{abstract}
Electricity prices are characterised by strong autoregressive persistence, periodicity (e.g. intraday, day-of-the week and month-of-the-year effects), large spikes or jumps, GARCH and -as evidenced by recent findings- periodic volatility. We propose a multivariate model of volatility that decomposes volatility multiplicatively into a non-stationary (e.g. periodic) part and a stationary part with log-GARCH dynamics. Since the model belongs to the log-GARCH class, the model is robust to spikes or jumps, allows for a rich variety of volatility dynamics without restrictive positivity constraints, can be estimated equation-by-equation by means of standard methods even in the presence of feedback, and allows for Dynamic Conditional Correlations (DCCs) that can -optionally- be estimated subsequent to the volatilities. We use the model to study the hourly day-ahead system prices at Nord Pool, and find extensive evidence of periodic volatility and volatility feedback. We also find that volatility is characterised by (positive) leverage in half of the hours, and that a DCC model provides a better fit of the conditional correlations than a Constant Conditional Correlation (CCC) model.
\end{abstract}

JEL Classification: C22, C32, C51, C58.

Keywords: Electricity prices, financial return, volatility, $A R C H$, exponential GARCH, log-GARCH, Multivariate GARCH, Dynamic Conditional Correlations, inverse leverage, Nord Pool.

\footnotetext{
*We are grateful to Juan Ignacio Peña and participants at ECOMFIN2016 (Paris) for useful comments, suggestions and questions

${ }^{\dagger}$ Department of Economics, Universidad Carlos III de Madrid (Spain).

‡ Corresponding author. Department of Economics, BI Norwegian Business School, Nydalsveien 37, 0484 Oslo, Norway. Email genaro.sucarrat@bi.no, phone $+47+46410779$, fax $+47+23264788$. Webpage: http://www.sucarrat.net/
} 
1 Introduction 2

2 Model and estimator $\quad 5$

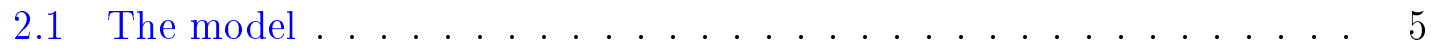

2.2 Equation-by-equation estimation . . . . . . . . . . . 6

2.3 Stochastic covariates ("X") . . . . . . . . . . . . 9

2.4 Dynamic Conditional Correlations (DCCs) . . . . . . . . . 9

3 The volatility of hourly day-ahead system prices at Nord Pool 10

3.1 Data . . . . . . . . . . . . . . 10

3.2 Models of the mean and volatility . . . . . . . . . . 11

3.3 Dynamic Conditional Correlations . . . . . . . . . . . 12

4 Conclusions $\quad 14$

$\begin{array}{ll}\text { References } & 14\end{array}$

\section{Introduction}

Modelling the uncertainty or volatility of electricity prices is of great importance for energy market participants. On the supply side, producers of electricity need estimates of the time-varying price volatility in order to determine the risks of future production levels. On the demand side, consumers of electricity need the same type of information in order to ascertain the risks associated with decisions about when and where to produce goods, and in order to hedge against adverse price changes.

It is well known that electricity prices are characterised by autoregressive persistence, periodicity effects (e.g. hour-of-the-day, day-of-the-week and month-of-the-year effects) in the conditional mean, see e.g. Bunn (2000), Knittel and Roberts (2005), Janczura et al. (2013), and Weron (2014). It is also well known that the volatility of electricity prices is characterised by Autoregressive Conditional Heteroscedasticity (ARCH) and large spikes or jumps, see e.g. Escribano et al. (2002, 2011), Koopman et al. (2007), and Hellström et al. (2012). Since the periodicity effects in the conditional mean usually account for a considerable proportion of the conditional mean dynamics, it is reasonable to conjecture that the same may also be the case for volatility. Recently, this line of research has received increasing attention. Bauwens et al. (2013, Section 4.2), for example, in a three-dimensional multivariate model of monthly, quarterly and yearly Phelix baseload futures at the European Energy Exchange, find that volatility depends on the number of days-to-delivery, i.e. that the volatility increases as the future in question approaches maturity. Sucarrat et al. (2016, Section 4), in a two-dimensional multivariate model of peak and off-peak day-ahead prices in the Oslo region (Nord Pool), find that day-of-the-week effects matter for volatility, and that peak volatility dynamics is less persistent than off- 
peak. Dupuis (2016), in a fifteen-dimensional multivariate model of electricity prices in the New York area, includes dummies in the volatility equations to accommodate hour-of-the-day and day-of-the-week effects.

There are two main challenges in the multivariate modelling of electricity price volatility. The first is the socalled "curse of dimensionality": As the multivariate dimension grows, joint estimation of the full model becomes infeasible in practice due to the number of parameters that has to be estimated. This problem is not specific to electricity prices, but it is more severe. The reason is that volatility is likely to depend on additional covariates, e.g. weather and market specific stochastic conditioning variables, in addition to periodicity effects similar to those that often characterise the conditional mean dynamics. Moreover, if standard or non-exponential GARCH models are used, then the curse of dimensionality problem is compounded, since the covariates and/or their parameters need to be restricted in estimation in order to ensure the positivity of fitted volatility. An example in which such a parameter restriction is needed in electricity price markets is the socalled "inverse leverage effect", as coined by Knittel and Roberts (2005), whereby negative shocks in one period leads to a reduction in volatility in the next period. ${ }^{1}$ Knittel and Roberts (2005) avoid the need for a restriction by using Nelson's (1991) Exponential GARCH (EGARCH). However, as is well-known, the EGARCH is not robust to spikes. ${ }^{2}$ This leads to the second main challenge in the modelling of electricity prices: The occurrence of price spikes. It is well-known that the ordinary GARCH model is not robust to such spikes. This is because the spikes affect estimation and inference inadvertently (Carnero et al. (2007), Gregory and Reeves (2010)), and because it makes the model propensive to volatility forecast failure subsequent to the spikes, see e.g. Harvey and Sucarrat (2014, Introduction). One multivariate model specification that has been put forward as being able to accommodate fat-tailed standardised errors, is the exponential version of the Generalised Autoregressive Score (GAS) model, see e.g. Creal et al. (2011). However, even univariate versions of this model can be very difficult to estimate due to its nature (see the section on "Computational challenges" in Sucarrat (2013, p. 142)), and the problem is compounded even further in the multivariate case.

We propose a multivariate model of electricity price volatility that is robust to spikes, that sidesteps the curse of dimensionality through equation-by-equation estimation, and which can include both deterministic and stochastic covariates to accommodate periodicity effects, leverage, the effect of weather-related variables, and so on. The model we propose is a multivariate multiplicative component logGARCH-X model that decomposes volatility multiplicatively into a non-stationary

\footnotetext{
${ }^{1}$ In stock markets, by contrast, a negative shock is usually followed by an increase. Arguably, the inverse leverage effect should instead be referred to as negative asymmetry, since the effect is not due to leverage in many markets (e.g. electricity and currency markets), and because a negative parameter value is not obtained as the mathematical inverse of a positive parameter.

${ }^{2}$ This is the reason why Nelson proposed his model in combination with the Generalised Error Distribution (GED) rather than with the standardised Student's $t$, since the unconditional variance will generally not exist if the standardised error is distributed as the latter, see Nelson (1991, p. $365)$.
} 
deterministic part of arbitrary form, and a stationary stochastic part. In order to enable equation-by-equation estimation, we make use of recent ideas developed formally in Francq and Zakoïan (2015), and in Francq and Sucarrat (2015). In particular, our model allows for feedback volatility effects among the equations, and Dynamic Conditional Correlations (DCCs) that - optionally - can be estimated subsequent to the volatility equations. As long as the DCC specification is appropriately chosen, this will ensure positive definiteness of the conditional covariance matrix. The model we propose can be viewed as a generalisation of Sucarrat et al. (2016, Section 4) in two ways. First, the deterministic component is much more general, since it can be of arbitrary form (i.e. it needs not be a linear combination of non-stochastic covariates). Second, we set up the estimation problem in such a way that the deterministic and stationary parts can be estimated separately, each by common methods that are widely available. In particular, in many cases the deterministic part will be estimable by an Ordinary Least Squares (OLS) regression, and the stochastic part will be estimable via an ARMA-regression. The equation-by-equation estimation procedure that we propose is thus readily implemented in software that is widely available. We use the model to study the multivariate volatility of hourly day-ahead system prices at Nord Pool. We find extensive evidence of periodicity in the volatility in that it depends on the day-of-the-week, and in that volatility dynamics varies intradaily. We also find extensive evidence of volatility feedback from adjacent hours. Leverage (of positive type), however, is only present in about half the instances, and it is at its strongest from $1 \mathrm{am}$ to $6 \mathrm{am}$. In only a single instance - at midnight - does a plain $\log$-GARCH(1,1) without periodicity provide a better fit of the volatility. Finally, we also find that the corrected DCC (cDCC) of Aielli (2013) provides a better fit of the conditional correlations than a Constant Conditional Correlation (CCC) specification. Interestingly, the conditional correlations are found to be at their strongest among adjacent hours, and that the strength is inversely related to the degree of adjacency: The further away, the weaker the correlation. This has implication for risk-management, since it implies that portfolios risk is reduced if the degree of adjacency among the portfolio components is reduced.

The rest of the paper is organised as follows. The next section, Section 2, outlines the model and the equation-by-equation estimation procedure. Section 3 contains our study of hourly day-ahead price volatility at Nord Pool. Section 4 contains the conclusions, whereas tables and figures are located at the end after the references. 


\section{Model and estimator}

\subsection{The model}

Let $\boldsymbol{r}_{t}=\left(r_{1 t}, \ldots, r_{M t}\right)^{\prime}$ denote an $M$-dimensional vector of price returns at $t$. A generic model of $\boldsymbol{r}_{t}$ can be written as (see e.g. Engle (2002))

$$
\begin{aligned}
\boldsymbol{r}_{t} & =\boldsymbol{\mu}_{t}+\boldsymbol{\epsilon}_{t}, \quad t \in \mathbb{Z}, \\
\boldsymbol{\epsilon}_{t} & =\left(\epsilon_{1 t}, \ldots, \epsilon_{M t}\right)^{\prime}, \quad \boldsymbol{H}_{t}=E_{t-1}\left(\boldsymbol{\epsilon}_{t} \boldsymbol{\epsilon}_{t}^{\prime}\right), \quad \boldsymbol{D}_{t}^{2}=\operatorname{diag}\left(\boldsymbol{H}_{t}\right), \\
\boldsymbol{\eta}_{t} & =\boldsymbol{D}_{t}^{-1} \boldsymbol{\epsilon}_{t}, \quad \boldsymbol{R}_{t}=E_{t-1}\left(\boldsymbol{\eta}_{t} \boldsymbol{\eta}_{t}^{\prime}\right),
\end{aligned}
$$

where $\boldsymbol{\mu}_{t}$ is the conditional mean (say, a VARMA-X), $\boldsymbol{\epsilon}_{t}=\left(\epsilon_{1 t}, \ldots, \epsilon_{M t}\right)^{\prime}$ is the error term, $\boldsymbol{H}_{t}$ is an $M \times M$ covariance matrix conditional on the past information set $\mathcal{F}_{t-1}, E_{t-1}(\cdot)$ is shorthand notation for $E\left(\cdot \mid \mathcal{F}_{t-1}\right), \boldsymbol{D}_{t}^{2}$ is a diagonal $M \times M$ matrix with the conditional variance or volatility $\boldsymbol{\sigma}_{t}^{2}=\left(\sigma_{1 t}^{2}, \ldots, \sigma_{M t}^{2}\right)^{\prime}$ on the diagonal, $\boldsymbol{\eta}_{t}=$ $\left(\eta_{1 t}, \ldots, \eta_{M t}\right)^{\prime}$ is the standardised error, i.e. $E\left(\boldsymbol{\eta}_{t}\right)=\mathbf{0}$ and $\operatorname{Var}\left(\boldsymbol{\eta}_{t}\right)=\mathbf{1}$ where $\mathbf{0}$ and $\mathbf{1}$ are $M \times 1$ vectors, $\boldsymbol{D}_{t}^{-1}$ is a diagonal $M \times M$ matrix with $\left(1 / \sigma_{1 t}, \ldots, 1 / \sigma_{M t}\right)^{\prime}$ on the diagonal and $\boldsymbol{R}_{t}$ is the correlation matrix conditional on the past. The relationships between $\boldsymbol{H}_{t}$ and $\boldsymbol{R}_{t}$ are given by $\boldsymbol{H}_{t}=\boldsymbol{D}_{t} \boldsymbol{R}_{t} \boldsymbol{D}_{t}$ and $\boldsymbol{R}_{t}=\boldsymbol{D}_{t}^{-1} \boldsymbol{H}_{t} \boldsymbol{D}_{t}^{-1}$. The return vector $\boldsymbol{r}_{t}$ can be replaced with a price vector $\boldsymbol{S}_{t}=\left(S_{1 t}, \ldots, S_{M t}\right)^{\prime}$, albeit - as is wellknown - any vector of prices can be obtained via a straightforward transformation of $\boldsymbol{r}_{t}$. For example, if $\boldsymbol{r}_{t}$ is log-return, then $\boldsymbol{S}_{t}=\exp \left(\boldsymbol{\mu}_{t}+\boldsymbol{\epsilon}_{t}\right) \odot \boldsymbol{S}_{t-1}$, where $\odot$ is the elementwise (Hadamard) matrix product. ${ }^{3}$ Alternatively, if $\boldsymbol{r}_{t}$ is relative return (this approach is preferable in markets where negative prices are possible), then $\boldsymbol{S}_{t}=\left(\boldsymbol{r}_{t}+\mathbf{1}\right) \odot \boldsymbol{S}_{t-1}$. Finally, to accommodate that our model belongs to the logGARCH class of models, and in order to enable equation-by-equation estimation, we need to add the two assumptions

$$
\begin{aligned}
& m=1, \ldots, M: P_{t-1}\left(\eta_{m t}=0\right)=0, \\
& \eta_{m t} \text { is independent of } \mathcal{F}_{t-1},
\end{aligned}
$$

where $P_{t-1}(\cdot)$ denotes a probability conditional on the past. The first assumption is standard in log-GARCH models, but can be relaxed via the modifications suggested in Sucarrat and Escribano (2013), and in Sucarrat and Grønneberg (2016). The second assumption enables equation-by-equation estimation of $\boldsymbol{\sigma}_{t}^{2}$ in the case where the conditional correlations (i.e. the off-diagonals of $\boldsymbol{R}_{t}$ ) are dynamic and dependent on the past, i.e. they are DCCs, see Francq and Zakoïan (2015), and Francq and Sucarrat (2015).

Periodic volatility means volatility is not covariance-stationary, since then the unconditional variance $E\left(\boldsymbol{\epsilon}_{t}^{2}\right)$ depends on $t$. The most common approach to nonstationary volatility is to decompose $\boldsymbol{\sigma}_{t}^{2}$ multiplicatively, see (amongst other) Van Bellegem and Von Sachs (2004), Engle and Rangel (2008), Mazur and Pipien (2012), and Amado and Terasvirta (2014a, 2014b). This means

$$
\boldsymbol{\sigma}_{t}^{2}=\boldsymbol{g}_{t} \odot \boldsymbol{h}_{t}=\left(g_{1 t} h_{1 t}, \ldots, g_{M t} h_{M t}\right)^{\prime},
$$

\footnotetext{
${ }^{3}$ For example, if $\boldsymbol{a}$ and $\boldsymbol{b}$ are two equally sized $M \times 1$ vectors, say, $\boldsymbol{a}=\left(a_{1}, \ldots, a_{M}\right)^{\prime}$ and $\boldsymbol{b}=\left(b_{1}, \ldots, b_{M}\right)^{\prime}$, then $\boldsymbol{a} \odot \boldsymbol{b}=\left(a_{1} b_{1}, \ldots, a_{M} b_{M}\right)^{\prime}$.
} 
where $\boldsymbol{g}_{t}$ is the non-stationary component and $\boldsymbol{h}_{t}$ is the stationary component (typically a GARCH-like process). In our model, the non-stationary component is specified as

$$
\ln \boldsymbol{g}_{t}=\left(\ln g_{1}\left(\boldsymbol{\lambda}_{1}, \boldsymbol{x}_{1 t}\right), \ldots, \ln g_{M}\left(\boldsymbol{\lambda}_{M}, \boldsymbol{x}_{M t}\right)\right)^{\prime},
$$

where $\ln g_{1}, \ldots, \ln g_{M}$ are known functions (linear or nonlinear), $\boldsymbol{x}_{1 t}, \ldots, \boldsymbol{x}_{M t}$ are known, non-stochastic regressors, and $\boldsymbol{\lambda}_{1}, \ldots, \boldsymbol{\lambda}_{M}$ are unknown parameters to be estimated. We do not restrict the $\boldsymbol{x}_{m t}$ 's nor the functions $\ln g_{m}$ to be equal across equations, and the $\ln g_{m}$ 's can assume a variety of shapes. In the simplest case the $\ln g_{m}$ 's are linear functions made up of time dummies (calendar effects, etc.), but it can also assume the shape of an exponential spline as in Engle and Rangel (2008), the Fourier Flexible Form (FFF) as in Mazur and Pipien (2012), or smooth threshold models as in Amado and Terasvirta (2014a, 2014b). Under appropriate assumptions, the functions may also be estimated nonparametrically, as in Van Bellegem and Von Sachs (2004).

The stationary component is specified as

$$
\ln \boldsymbol{h}_{t}=\boldsymbol{\omega}+\sum_{i=1}^{p} \boldsymbol{\alpha}_{i} \ln \widetilde{\boldsymbol{\epsilon}}_{t-i}^{2}+\sum_{j=1}^{q} \boldsymbol{\beta}_{j} \ln \boldsymbol{h}_{t-j}
$$

where $\ln \boldsymbol{h}_{t}=\left(\ln h_{1, t}, \ldots, \ln h_{M, t}\right)^{\prime}, \boldsymbol{\omega}=\left(\omega_{1}, \ldots, \omega_{M}\right)^{\prime}, \ln \widetilde{\boldsymbol{\epsilon}}_{t}^{2}=\left(\ln \boldsymbol{\epsilon}_{t}^{2}-\ln \boldsymbol{g}_{t}\right)=$ $\left(\ln h_{1 t} \eta_{1 t}^{2}, \ldots, \ln h_{M t} \eta_{M t}^{2}\right)^{\prime}$,

$$
\boldsymbol{\alpha}_{i}=\left(\begin{array}{ccc}
\alpha_{11 . i} & \cdots & \alpha_{1 M . i} \\
\vdots & \ddots & \vdots \\
\alpha_{M 1 . i} & \cdots & \alpha_{M M . i}
\end{array}\right) \quad \text { and } \quad \boldsymbol{\beta}_{j}=\operatorname{diag}\left(\beta_{11 . j}, \ldots, \beta_{M M . j}\right) .
$$

The non-diagonality of $\boldsymbol{\alpha}_{i}$ enables feedback among equations, whereas the diagonality of $\boldsymbol{\beta}_{j}$ enables equation-by-equation estimation. The model is stable (in $\ln \boldsymbol{h}_{t}$ ) if all eigenvalues of $\sum_{i=1}^{p}\left(\boldsymbol{\alpha}_{i}+\boldsymbol{\beta}_{i}\right)$ are strictly less than 1 in modulus, and $\ln \boldsymbol{h}_{t}$ is invertible if all the eigenvalues of $\sum_{j=1}^{q} \boldsymbol{\beta}_{j}$ are strictly less than 1 in modulus.

\subsection{Equation-by-equation estimation}

In our model, given by (1)-(8), the $m$ th. log-volatility equation can be written as

$$
\begin{aligned}
\ln \sigma_{m t}^{2} & =\ln g_{m t}+\ln h_{m t}, \\
\ln g_{m t} & =\ln g_{m}\left(\boldsymbol{\lambda}_{m}, \boldsymbol{x}_{m t}\right), \\
\ln h_{m t} & =\omega_{m}+\sum_{i=1}^{p} \boldsymbol{\alpha}_{m . i} \ln \widetilde{\boldsymbol{\epsilon}}_{t-i}^{2}+\sum_{j=1}^{q} \beta_{m m . j} \ln \sigma_{m, t-j}^{2},
\end{aligned}
$$

where $\boldsymbol{\alpha}_{m . i}$ is the $m$ th. row of $\boldsymbol{\alpha}_{i}$, i.e. $\boldsymbol{\alpha}_{m . i}=\left(\alpha_{m 1 . i}, \ldots, \alpha_{m M . i}\right)$. Estimation of equation $m$ proceeds in three steps: 
1. Estimate $\boldsymbol{\lambda}_{m}$ by means of the auxiliary regression

$$
\ln \epsilon_{m t}^{2}=\lambda_{m 0}+\ln g_{m}\left(\boldsymbol{\lambda}_{m}, \boldsymbol{x}_{m t}\right)+y_{m t},
$$

where $\lambda_{m 0}$ is the intercept and $y_{m t}$ the error-term. Below, we show that $\lambda_{m 0}=E\left(\ln \widetilde{\epsilon}_{m t}^{2}\right)$ and that $y_{m t}$ is a zero-mean stationary error under standard assumptions. In the case where $\boldsymbol{\lambda}_{m}$ enters linearly in $\ln g_{m}$ the parameters can be estimated by OLS.

2. Fit an ARMA model to the residuals $\widehat{y}_{m t}$ from the first step. Below, we show that the error-term $y_{m t}$ from Step 1 is in fact governed by a meancorrected ARMA representation of $\ln h_{m t}$. Due to the relationships between the parameters of the log-GARCH model and the parameters of the meancorrected ARMA-representation, this provides consistent estimates of all the log-GARCH parameters apart from the intercept $\omega_{m}$. As we will show, however, an estimate of $\omega_{m}$ is not needed in order to estimate $\sigma_{m t}^{2}$. Nevertheless, $\omega_{m}$ can - if needed - be estimated subsequently in a fourth step (see below).

3. Estimate the $\log$-moment $E\left(\ln \eta_{m t}^{2}\right)$ needed to complete the estimate of $\sigma_{m t}^{2}$. As we show below, estimation of $E\left(\ln \eta_{m t}^{2}\right)$ is straightforward by means of a simple formula made up of the residuals from Step 2.

We now provide the details of this three step estimator.

Step 1 consists of estimating an auxiliary regression whose error-term follows the mean-corrected ARMA-representation of $\ln h_{m t}$. If $E\left|\ln \eta_{m t}^{2}\right|<\infty$, then the ARMA-representation of $\ln h_{m t}$ is

$$
\ln \widetilde{\epsilon}_{m t}^{2}=\phi_{m 0}+\sum_{i=1}^{p} \phi_{m . i} \ln \widetilde{\boldsymbol{\epsilon}}_{t-i}^{2}+\sum_{j=1}^{q} \theta_{m m . j} u_{m, t-j}+u_{m, t},
$$

where

$$
\begin{aligned}
& u_{m, t}=\ln \eta_{m, t}^{2}-E\left(\ln \eta_{m, t}^{2}\right), \quad u_{m, t} \sim W N\left(0, \sigma_{u_{m}}^{2}\right) \\
& \phi_{m 0}=\omega_{m}+\left(1-\sum_{j=1}^{q} \beta_{m m . j}\right) E\left(\ln \eta_{m t}^{2}\right) \\
& \phi_{m . i}=\left(\alpha_{m 1 . i}, \ldots, \alpha_{m m . i}+\beta_{m m . i}, \ldots, \alpha_{m M . i}\right) \quad \text { and } \quad \theta_{m m . j}=-\beta_{m m . j} .
\end{aligned}
$$

In other words, $\phi_{m . i}$ is the $m$ th. row of $\phi_{i}$. If $E\left|\ln \widetilde{\boldsymbol{\epsilon}}_{t}^{2}\right|<\infty$, then the mean-corrected ARMA representation is

$$
y_{m, t}=\sum_{i=1}^{p} \boldsymbol{\phi}_{m . i} \boldsymbol{y}_{t-i}+\sum_{j=1}^{q} \theta_{m m . j} u_{m, t-j}+u_{m, t} \quad u_{m, t} \sim W N\left(0, \sigma_{u_{m}}^{2}\right),
$$

where $y_{m, t}=\ln \widetilde{\epsilon}_{m, t}^{2}-E\left(\ln \widetilde{\epsilon}_{m, t}^{2}\right)$ and $\boldsymbol{y}_{t}=\left(y_{1, t}, \ldots, y_{M, t}\right)^{\prime}$. To obtain the auxiliary regression in (18), we simply add $\ln \eta_{m t}^{2}$ to $\ln \sigma_{m t}^{2}$ in (9), which gives

$$
\begin{aligned}
\ln \epsilon_{m t}^{2} & =\ln g_{m t}+\ln h_{m t}+\ln \eta_{m t}^{2} \\
& =\ln g_{m t}+\ln \widetilde{\epsilon}_{m t}^{2} \\
& =\lambda_{m 0}+\ln g_{m t}+y_{m t},
\end{aligned}
$$


where $\lambda_{m 0}=E\left(\ln \widetilde{\epsilon}_{m t}^{2}\right)$ and $y_{m t}=\ln \widetilde{\epsilon}_{m t}^{2}-E\left(\ln \widetilde{\epsilon}_{m t}^{2}\right)$. In other words, (18) is a standard regression model in which the error-term follows a zero-mean stationary error. In particular, if $\boldsymbol{\lambda}_{m}$ enters $\ln g_{m t}$ linearly, then $\lambda_{m 0}+\ln g_{m t}$ can be estimated by OLS.

Step 2 consists of estimating (17) using the residuals $\widehat{y}_{m t}$ from Step 1 . This is an ARMA-X estimation problem that provides estimates of all the ARCH and GARCH parameters - except $\omega_{m}$-due to the relationships in (16). An estimate of $\omega_{m}$, however, is not needed if the aim is to estimate $\sigma_{m t}^{2}$. The reason for this is that the fitted values from the first two steps provide estimates of $E\left(\ln \widetilde{\epsilon}_{m t}^{2}\right)+\ln g_{m t}$ and $E_{t-1}\left(y_{m t}\right)$, respectively. Adding these gives

$$
\begin{aligned}
E\left(\ln \widetilde{\epsilon}_{m t}^{2}\right)+\ln g_{m t}+E_{t-1}\left(y_{m t}\right) & =\ln g_{m t}+E_{t-1}\left(\ln \widetilde{\epsilon}_{m t}^{2}\right) \\
& =\ln g_{m t}+\ln h_{t}+E\left(\ln \eta_{m t}^{2}\right),
\end{aligned}
$$

since $\ln \widetilde{\epsilon}_{m t}^{2}=\ln h_{m t}+\ln \eta_{m t}^{2}$. So only an estimate of $E\left(\ln \eta_{m t}^{2}\right)$ is needed to complete the estimate of $\sigma_{m t}^{2}$.

Step 3 thus consists of estimating $E\left(\ln \eta_{m t}^{2}\right)$. Sucarrat et al. (2016) noted that, if $E\left|\ln \eta_{m t}^{2}\right|<\infty$ and $E\left(\eta_{m t}^{2}\right)=1$, then it follows straightforwardly that $-\ln E\left(\exp \left(u_{m t}\right)\right)=$ $E\left(\ln \eta_{m t}^{2}\right)$. This suggests

$$
-\ln \left[T^{-1} \sum_{t=1}^{T} \exp \left(\widehat{u}_{m t}\right)\right]
$$

provides a consistent estimator of $E\left(\ln \eta_{m t}^{2}\right)$, where the $\widehat{u}_{m t}$ 's are the residuals from Step 2. ${ }^{4}$ Sucarrat et al. (2016) provide conditions under which this indeed holds, whereas Francq and Sucarrat (2015) prove that this holds when the ARMA-X representation of equation $m$ in a first order multivariate log-GARCH-X model - where the X-part refers to stochastic conditioning variables - is estimated by Least Squares.

Summarised, then, the estimate of $\sigma_{m t}^{2}$ is given by

$$
\widehat{\sigma}_{m t}^{2}=\exp \left(\widehat{E}\left(\ln \widetilde{\epsilon}_{m t}^{2}\right)+\ln \widehat{g}_{m t}+\widehat{E}_{t-1}\left(y_{m t}\right)-\widehat{E}\left(\ln \eta_{m t}^{2}\right)\right)
$$

where $\widehat{E}\left(\ln \widetilde{\epsilon}_{m t}^{2}\right)+\ln \widehat{g}_{m t}$ is the fitted value of the auxiliary regression in Step 1 , $\widehat{E}_{t-1}\left(y_{m t}\right)$ is the fitted value of the mean-corrected ARMA representation in Step 2 and $\widehat{E}\left(\ln \eta_{m t}^{2}\right)$ is the estimate of $E\left(\ln \eta_{m t}^{2}\right)$ in Step 3 .

An estimate of $\omega_{m}$ requires estimation of the other equations, in addition to equation $m$. This is because the expression for $E\left(\ln \widetilde{\epsilon}_{m t}^{2}\right)$, which can be written as $E\left(\ln \widetilde{\epsilon}_{m t}^{2}\right)=\phi_{m 0}+\sum_{i=1}^{p} \phi_{m . i} E\left(\ln \widetilde{\boldsymbol{\epsilon}}_{t}^{2}\right)$, depends on the unconditional expectations of the other equations. Solving for $\omega_{m}$ in the expression for $E\left(\ln \widetilde{\epsilon}_{m t}^{2}\right)$ gives

$$
\omega_{m}=\left(1-\sum_{j=1}^{q} \beta_{m m . j}\right) E\left(\ln \widetilde{\epsilon}_{m t}^{2}\right)-\sum_{i=1}^{p} \boldsymbol{\alpha}_{m . i} E\left(\ln \widetilde{\boldsymbol{\epsilon}}_{t}^{2}\right)-\left(1-\sum_{j=1}^{q} \beta_{m m . j}\right) E\left(\ln \eta_{m t}^{2}\right),
$$

\footnotetext{
${ }^{4}$ The expression in square brackets in (20) is the smearing estimate of Duan (1983).
} 
where we have used the expression for $\phi_{m 0}$ in (15), and that $\sum_{i=1}^{p} \phi_{m . i} E\left(\ln \widetilde{\boldsymbol{\epsilon}}_{t}^{2}\right)=$ $\sum_{i=1}^{p} \boldsymbol{\alpha}_{m . i} E\left(\ln \widetilde{\boldsymbol{\epsilon}}_{t}^{2}\right)+\sum_{j=1}^{q} \beta_{m m . j} E\left(\ln \widetilde{\epsilon}_{m t}^{2}\right)$. It should be noted that only the elements in $E\left(\ln \widetilde{\boldsymbol{\epsilon}}_{t}^{2}\right)$, apart from the $m$ th. entry, comes from the other equations. In other words, if there is no feedback effects (i.e. all entries in the $\boldsymbol{\alpha}_{m . i}$ 's apart from the $m$ th. entry are zero), then there is no need to estimate the other equations in order to estimate $\omega_{m}$.

\subsection{Stochastic covariates ("X")}

Electricity price volatility is likely to depend on a range of stochastic factors, e.g. leverage, weather-related quantities and other market specific variables. An appealing property of the model and associated estimation procedure described above is therefore that the stationary component $\boldsymbol{h}_{t}$ can straightforwardly be augmented by stationary stochastic covariates, "X". Let $\boldsymbol{x}_{t}^{s}=\left(x_{1 t}^{s}, x_{2 t}^{s}, \ldots\right)^{\prime}$ denote a vector of covariance-stationary variables, where the superscript $s$ is intended to connote "stochastic" (in contrast to the non-stochastic regressors in the non-stationary component). For notational convenience, but without any loss of generality, we henceforth assume that $\boldsymbol{x}_{t}^{s}$ is mean-corrected, so that $E\left(\boldsymbol{x}_{t}^{s}\right)=0$. The stationary component in (8) now becomes

$$
\ln \boldsymbol{h}_{t}^{2}=\boldsymbol{\omega}+\sum_{i=1}^{p} \boldsymbol{\alpha}_{i} \ln \widetilde{\boldsymbol{\epsilon}}_{t-i}^{2}+\sum_{j=1}^{q} \boldsymbol{\beta}_{j} \ln \boldsymbol{h}_{t-j}^{2}+\boldsymbol{\delta} \boldsymbol{x}_{t-1}^{s},
$$

where $\boldsymbol{\delta}$ is a parameter-matrix of appropriate size. Contrary to non-exponential GARCH models, we do not need to impose any non-negativity constraints on $\boldsymbol{\delta}$, nor on the variables in $\boldsymbol{x}_{t}^{s}$. The ARMA-representation of $\ln h_{m t}$ is now

$$
\ln \widetilde{\epsilon}_{m t}^{2}=\phi_{m 0}+\sum_{i=1}^{p} \boldsymbol{\phi}_{m . i} \ln \widetilde{\boldsymbol{\epsilon}}_{t-i}^{2}+\sum_{j=1}^{q} \theta_{m m . j} u_{m, t-j}+\boldsymbol{\delta}_{m} \boldsymbol{x}_{t-1}^{s}+u_{m, t},
$$

where $\boldsymbol{\delta}_{m}$ is the $m$ th. row of $\boldsymbol{\delta}$, and where $\phi_{m 0}, \phi_{m . i}, \theta_{m m . j}$ and $u_{m t}$ are the same as before, i.e. they are given by (14)-(16). The mean-corrected ARMA representation now becomes

$$
y_{m, t}=\sum_{i=1}^{p} \boldsymbol{\phi}_{m . i} \boldsymbol{y}_{t-i}+\sum_{j=1}^{q} \theta_{m m . j} u_{m, t-j}+\boldsymbol{\delta}_{m} \boldsymbol{x}_{t-1}^{s}+u_{m, t},
$$

where $y_{m, t}, \boldsymbol{y}_{t}$ and $u_{m t}$ are defined as earlier. The practical consequence of this is that the three step estimation procedure described above only requires one minor modification: Estimate (25) instead of (17) in Step 2. The other steps are unchanged, and if an estimate of $\omega_{m}$ is needed, then formula (22) can still be used.

\subsection{Dynamic Conditional Correlations (DCCs)}

Assumption (5) enables DCCs. The three-step estimation procedure described above, however, does not provide estimates of the DCCs. Nevertheless, they can - 
if needed - be estimated in a subsequent step. The estimates $\widehat{\sigma}_{1 t}^{2}, \ldots, \widehat{\sigma}_{M t}^{2}$ from the three-step procedure above lead to the standardised residuals $\widehat{\boldsymbol{\eta}}_{t}=\left(\widehat{\eta}_{1 t}, \ldots, \widehat{\eta}_{M t}\right)^{\prime}$, where $\widehat{\eta}_{m t}=\epsilon_{m t} / \widehat{\sigma}_{m t}$. These residuals can be used to estimate a DCC specification of $\boldsymbol{R}_{t}=E\left(\boldsymbol{\eta}_{t} \boldsymbol{\eta}_{t}^{\prime} \mid \mathcal{F}_{t-1}\right)$. An example is the DCC of Engle (2002), or alternatively the corrected version of Aielli (2013), see e.g. the empirical section of Francq and Sucarrat (2015). Another option is the robust (to spikes) DCC model proposed for electricity prices by Dupuis (2016).

\section{The volatility of hourly day-ahead system prices at Nord Pool}

\subsection{Data}

Nord Pool Spot AS is one of the largest energy exchanges in the world measured in traded volume of terrawatts per hour (TWh). Currently 380 members operate on the exchange, and these include public and private energy producers, energy intensive industries, large consumers, distributors, funds, investment companies, banks, brokers, utility companies and financial institutions. Arguably, the most important price at the exchange is the "system price". This is because it constitutes some sort of aggregate or equilibrium price (it is determined by the intersection of the aggregate supply and demand curves of all bids and offers), and because it is used as reference in financial contracts - used for hedging and risk management traded at Nasdaq Commodities. ${ }^{5}$

Our rawdata consist of the hourly day-ahead system prices in Euros per $\mathrm{kw} / \mathrm{h}$ from 1 January 2010 to 20 May 2014. This amounts to $T=1601$ daily observations for each $m$ before differencing and lagging. The price at day $t$ in hour $m$ we denote $S_{m t}$, where $m=1, \ldots, 24$. Note that $S_{1 t}$ should be interpreted as the price from midnight to 1 am in day $t, S_{2 t}$ is the price from 1am to 2 am in day $t$, and so on. The daily log-return for hour $m$, denoted $r_{m t}$, is defined as $\ln S_{m, t}-\ln S_{m, t-1}$, i.e. the daily log-return for hour $m$. Graphs of $S_{m t}$ and $r_{m t}$ are contained in Figures 1 and 2, whereas the top graph in Figure 3. The prices and returns exhibit the usual characteristics, i.e. that price variability is substantially larger than those of stocks, stock indices and exchange rates, and that big spikes or jumps occur relatively frequently. On average, the price is highest at $9 \mathrm{am}$ and lowest at $4 \mathrm{am}$. There are no negative prices in our data, but five spurious zeros due to daylight saving time. ${ }^{6}$ These zeros we replace by the average of the two adjacent values.

\footnotetext{
${ }^{5}$ See https://www.nordpoolspot.com/About-us/, http://www.nordpoolspot.com/ How-does-it-work/Financial-market/ and http://ww. nordpoolspot.com/TAS/ Day-ahead-market-Elspot/Price-calculation/. All accessed 14 July 2016.

${ }^{6}$ The five zeros all occurred for $m=3$, one in each year: 28 March 2010, 27 March 2011, 25 March 2012, 31 March 2013 and 30 March 2014.
} 


\subsection{Models of the mean and volatility}

We start by fitting a conditional mean specification to the vector $\boldsymbol{r}_{t}$ of daily returns. A restricted Vector Autoregressive (VAR) model is formulated and estimated, where each equation contains its own AR-lags from 1 to 7 and daily impulse dummies (Tuesday to Sunday). The total number of estimated parameters in each equation is thus fourteen: One intercept + seven AR-parameters + six dummy-parameters. The second column of Table 1 and the second graph in Figure 3 contain the $R$ squareds of the twenty-four conditional mean equations. As is clear, predictability varies substantially across the day, since the $R$-squareds range from only $5 \%$ when $m=1$ to a peak of $50 \%$ when $m=9$. As a whole, the graph clearly indicates that the explanatory power is higher in peak hours, i.e. from about $m=7$ to about $m=19$.

We fit five different multivariate volatility models to the vector of errors $\boldsymbol{\epsilon}_{t}$. The models we label (a) - (e), and in each of the five models equation $m$ is given by

$$
\begin{aligned}
\text { (a) } \ln \sigma_{m t}^{2}= & \omega+\alpha_{m m .1} \ln \widetilde{\epsilon}_{m, t-1}^{2}+\beta_{m m .1} \ln h_{m, t-1}, \\
\text { (b) } \ln \sigma_{m t}^{2}= & \sum_{i=1}^{6} \lambda_{m i} d_{i t}, \\
\text { (c) } \ln \sigma_{m t}^{2}= & \sum_{i=1}^{6} \lambda_{m i} d_{i t}+\omega+\alpha_{m m .1} \ln \widetilde{\epsilon}_{m, t-1}^{2}+\beta_{m m .1} \ln h_{m, t-1}, \\
\text { (d) } \ln \sigma_{m t}^{2}= & \sum_{i=1}^{6} \lambda_{m i} d_{i t}+\omega+\alpha_{m m .1} \ln \widetilde{\epsilon}_{m, t-1}^{2}+\alpha_{m m(1) .1} \ln \widetilde{\epsilon}_{m(1), t-1}^{2} \\
& +\alpha_{m m(2) .1} \ln \widetilde{\epsilon}_{m(2), t-1}^{2}+\beta_{m m .1} \ln h_{m, t-1}, \\
\text { (e) } \ln \sigma_{m t}^{2}= & \sum_{i=1}^{6} \lambda_{m i} d_{i t}+\omega+\alpha_{m m .1} \ln \widetilde{\epsilon}_{m, t-1}^{2}+\alpha_{m m(1) .1} \ln \widetilde{\epsilon}_{m(1), t-1}^{2} \\
& +\alpha_{m m(2) .1} \ln \widetilde{\epsilon}_{m(2), t-1}^{2}+\beta_{m m .1} \ln h_{m, t-1}+\delta_{m} x_{m, t-1} .
\end{aligned}
$$

Model (a) is a plain $\log$-GARCH $(1,1)$ and serves as benchmark. The variables $d_{1 t}, \ldots, d_{6 t}$ are dummies for Tuesday to Sunday, respectively. So (b)-(e) all contain periodicity. In (e) and (d), $\ln \widetilde{\epsilon}_{m(1), t-1}^{2}$ and $\ln \widetilde{\epsilon}_{m(2), t-1}^{2}$ are the two most adjacent $\log$-ARCH lags in equation $m$. For example, if $m=1$, then $m(1)=2$ and $m(2)=3$. Similarly, if $m=2$, then $m(1)=m-1=1$ and $m(2)=m+1=3$. And so on. The idea is to include those $\log$ - $\mathrm{ARCH}$ terms that are most likely to have a feedback effect on the volatility of equation $m$. Finally, the variable $x_{m, t-1}$ is a lagged meancorrected asymmetry or "leverage" term, where the lagged asymmetry term is given by $I_{\left\{\epsilon_{m, t-1}<0\right\}}$.

Estimation of the five multivariate models all together takes about thirty seconds on an average laptop, and we experience no numerical issues. ${ }^{7}$ Both the deterministic and stationary parts are estimated by Least Squares (LS), and a summary of the

\footnotetext{
${ }^{7}$ The computations were undertaken with $R$ code on a Lenovo X250 with an Intel Core i7$5600 \mathrm{U}-2.60 \mathrm{Ghz}$ processor running Win7-64bit.
} 
estimation results are contained in Table 1. For comparison we use the Schwarz (1978) information criterion (BIC), which favours parsimony. The best model in hour $m$ according to the BIC is identified with an asterisk $\left(^{*}\right)$ to the right of its BICvalue. In all but one case the best model is either $(\mathrm{d})$ or $(\mathrm{e})$. In other words, in all but one case the best model contains periodicity and feedback terms. The exception occurs at midnight, i.e. for $m=24$, in which model (a) is the best according to the BIC. If we only compare (a), (b) and (c) against each other to obtain a more detailed idea of the importance of periodicity, then we see that either (b) or (c) performs better in 17 out of the 24 hours. Moreover, the periods in which (a) performs better are clustered in the evening, since they are $m=17,18,19,20,21,22$ and 24. Finally, with respect to leverage, equation (e) performs better than (d) in 12 instances, whereas the opposite is the case in 11 instances. In other words, there is evidence of leverage in about half of the hours. Interestingly, whenever present, the leverage is always positive - i.e. we find no evidence of the socalled inverse (i.e. negative) leverage effect, and most of the instances of leverage occurs at night from $m=21$ to $m=6$. Summarised, then, our results provides extensive evidence of periodicity and feedback effects in the volatility, and in about half of the hours there is leverage. In only a single instance, at midnight, did the plain $\log -\operatorname{GARCH}(1,1)$ perform better than the other specifications.

The third, fourth and fifth graphs in Figure 3 provide a more detailed picture of the best specifications in each $m$. The third graph contains the ARCH(1) estimates, i.e. $\widehat{\alpha}_{m m .1}$, for $m=1, \ldots, 24$. All estimates but one are positive, and most are between 0 and 0.10 . In other words, once periodicity and feedback effects are controlled for, then the (own) ARCH effect becomes substantially smaller than commonly found in electricity prices, and much closer to those usually found in stock and currency markets. The fourth graph in the figure contains the GARCH(1) parameter

estimates, i.e. $\widehat{\beta}_{m m .1}$. Most of the values are in the 0.7 to 0.8 range, and interestingly the values below 0.7 are clustered around the morning hours, i.e. $m=5,6$ and 7 . Finally, the bottom graph contains the leverage estimates, i.e. $\widehat{\delta}_{m}$. The estimated value is zero if the best model in hour $m$ does not contain leverage, and it is notable that the strongest impact is clustered at night, from $m=1$ to $m=6$. Thereafter, leverage is either zero or very small.

\subsection{Dynamic Conditional Correlations}

To obtain estimates of the off-diagonals of $\boldsymbol{H}_{t}$, an estimate of $\boldsymbol{R}_{t}=E_{t-1}\left(\boldsymbol{\eta}_{t}^{\prime} \boldsymbol{\eta}_{t}\right)$ is needed. To this end we fit the corrected DCC (cDCC) model of Aielli (2013), which is a modified version of Engle's (2002) DCC. It should be noted that the cDCC is a covariance-stationary model of $\boldsymbol{\eta}_{t}^{\prime} \boldsymbol{\eta}_{t}$ even though $\boldsymbol{\epsilon}_{t}^{\prime} \boldsymbol{\epsilon}_{t}$ is not covariance stationary.

The cDCC model is given by

$$
\boldsymbol{R}_{t}=\boldsymbol{Q}_{t}^{*-1 / 2} \boldsymbol{Q}_{t} \boldsymbol{Q}_{t}^{*-1 / 2}, \quad \boldsymbol{Q}_{t}=\left(1-\gamma_{1}-\gamma_{2}\right) \boldsymbol{R}+\gamma_{1} \boldsymbol{Q}_{t-1}^{* 1 / 2} \boldsymbol{\eta}_{t-1} \boldsymbol{\eta}_{t-1}^{\prime} \boldsymbol{Q}_{t-1}^{* 1 / 2}+\gamma_{2} \boldsymbol{Q}_{t-1}
$$

where $\gamma_{1}, \gamma_{2} \geq 0$ are scalars such that $\gamma_{1}+\gamma_{2}<1, \boldsymbol{R}$ is a correlation matrix (which in general is not equal to $\left.E\left(\boldsymbol{R}_{t}\right)\right), \boldsymbol{Q}_{t}^{*}$ is a diagonal matrix with elements from the 
diagonal of $\boldsymbol{Q}_{t}$ and $\boldsymbol{\eta}_{t}=\boldsymbol{D}_{t}^{-1} \boldsymbol{\epsilon}_{t}$. Here, we define the standardised error $\boldsymbol{\eta}_{t}$ to be made up of the errors from the best model in each $m$ according to the BIC (see above). In other words, if $m=1$, then $\widehat{\eta}_{1 t}$ is that of model (e). If $m=8$, then $\widehat{\eta}_{8 t}$ is that of model (d). And so on.

Recalling that $\boldsymbol{R}_{t}$ is both the conditional correlation and covariance matrix of $\boldsymbol{\eta}_{t}$, estimation of $\gamma_{1}$ and $\gamma_{2}$ by Gaussian Quasi Maximum Likelihood (QML) leads to the estimator

$$
\left(\widehat{\gamma}_{1}, \widehat{\gamma}_{2}\right)=\arg \max _{\left(\widehat{\gamma}_{1}, \widehat{\gamma}_{2}\right)} \sum_{t=1}^{T}\left(-M \ln 2 \pi-\ln \left|\widehat{\boldsymbol{R}}_{t}\right|-\widehat{\boldsymbol{\eta}}_{t}^{\prime} \widehat{\boldsymbol{R}}_{t}^{-1} \widehat{\boldsymbol{\eta}}_{t}\right) / 2,
$$

where $\left|\boldsymbol{R}_{t}\right|$ is the determinant of $\boldsymbol{R}_{t}, \widehat{\boldsymbol{\eta}}_{t}$ are the standardised residuals of the best models,

$$
\begin{aligned}
& \widehat{\boldsymbol{R}}_{t}=\widehat{\boldsymbol{Q}}_{t}^{*-1 / 2} \widehat{\boldsymbol{Q}}_{t} \widehat{\boldsymbol{Q}}_{t}^{*-1 / 2}, \quad \widehat{\boldsymbol{Q}}_{t}=\left(1-\widehat{\gamma}_{1}-\widehat{\gamma}_{2}\right) \widehat{\boldsymbol{R}}+\widehat{\gamma}_{1} \widehat{\boldsymbol{Q}}_{t-1}^{* 1 / 2} \widehat{\boldsymbol{\eta}}_{t-1} \widehat{\boldsymbol{\eta}}_{t-1}^{\prime} \widehat{\boldsymbol{Q}}_{t-1}^{* 1 / 2}+\widehat{\gamma}_{2} \widehat{\boldsymbol{Q}}_{t-1} \\
& \widehat{\boldsymbol{R}}=\frac{1}{T} \sum_{t=1}^{T} \widehat{\boldsymbol{Q}}_{t}^{* 1 / 2} \widehat{\boldsymbol{\eta}}_{t} \widehat{\boldsymbol{\eta}}_{t}^{\prime} \widehat{\boldsymbol{Q}}_{t}^{* 1 / 2}, \quad \widehat{\boldsymbol{Q}}_{t}^{*}=\operatorname{diag}\left(\widehat{q}_{11 t}, \ldots, \widehat{q}_{M M t}\right) \\
& \widehat{q}_{m m t}=\left(1-\widehat{\gamma}_{1}-\widehat{\gamma}_{2}\right)+\widehat{\gamma}_{1} \widehat{\eta}_{m, t-1}^{2}+\widehat{\gamma}_{2} \widehat{q}_{m m, t-1} \quad \text { for } m=1, \ldots, M .
\end{aligned}
$$

The estimates of $\gamma_{1}$ and $\gamma_{2}$ are 0.004 and 0.905 , respectively, which suggests the correlations are relatively persistent. An estimate of the unconditional correlation $E\left(\boldsymbol{R}_{t}\right)$ is obtained as $\widehat{\boldsymbol{R}}^{*-1 / 2} \widehat{\boldsymbol{R}} \widehat{\boldsymbol{R}}^{*-1 / 2}$, where $\widehat{\boldsymbol{R}}^{*}$ is a diagonal matrix containing the diagonal elements of $\widehat{\boldsymbol{R}}$. Figure 4 depicts the evolution of the unconditional correlations over the day depending on $m$. The general tendency is clear: The strongest unconditional correlations of $\hat{E}\left(\eta_{i t} \eta_{m t}\right)$ are always those closest to hour $i$. The top left graph, for example, depicts the evolution of $\widehat{E}\left(\eta_{1 t} \eta_{m t}\right)$ for $m=1, \ldots 24$. Naturally, when $m=1$, then $\widehat{E}\left(\eta_{1 t} \eta_{1 t}\right)=1$. Next, the unconditional correlations fall gradually until they reach their lowest point at $m=23$, in which the estimate is 0.2 . In other words, almost without exception $\widehat{E}\left(\eta_{i t} \eta_{m+1, t}\right)$ is stronger than $\widehat{E}\left(\eta_{i t} \eta_{m+2, t}\right)$, which is stronger than $\widehat{E}\left(\eta_{i t} \eta_{m+3, t}\right)$, and so on. Similarly, whenever $i>1, \widehat{E}\left(\eta_{i t} \eta_{m-1, t}\right)$ is stronger than $\widehat{E}\left(\eta_{i t} \eta_{m-2, t}\right)$, which is stronger than $\widehat{E}\left(\eta_{i t} \eta_{m-3, t}\right)$, and so on. So just as in the case of volatility feedback (see above), there is a clear adjacency effect among correlations.

Estimation of the cDCC entails fitting a total of $24 \cdot(24-1) / 2=276$ distinct conditional correlation paths. Figure 5 contains graphs of the first 24 , i.e. of $\widehat{E}_{t-1}\left(\eta_{1 t} \eta_{m t}\right)$ for $m=1, \ldots, 24$. Graphically, they appear relatively flat around their unconditional values, so one may ask whether the cDCC provides a better fit than a Constant Conditional Correlation (CCC) specification. The Gaussian loglikelihood of the cDCC is given by the formula in (27) at the estimated values $\widehat{\gamma}_{1}$, $\widehat{\gamma}_{2}$. Similarly, the log-likelihood of the CCC specification is obtained by replacing $\widehat{\boldsymbol{R}}_{t}$ with the sample covariance matrix of $\widehat{\boldsymbol{\eta}}_{t} \widehat{\boldsymbol{\eta}}_{t}^{\prime}$ in the same formula. The two values are -20725.7 (cDCC) and -20938.0 (CCC), so the former produces a better fit in terms of the (quasi) log-likelihood. In terms of the BIC, computed in terms of the average 
(quasi) log-likelihood with $T=1592$, and defining the cDCC to be characterised by 2 parameters and the CCC by 0 , then this yields BIC values of 26.05 (cDCC) and 26.30 (CCC), respectively. In other words, the DCC specification is also warranted according to the BIC.

\section{Conclusions}

We propose a multivariate model of electricity price volatility that decomposes volatility multiplicatively into a non-stationary part (e.g. periodic) of arbitrary form, and a stationary part with log-GARCH dynamics. The model is robust to spikes or jumps, a common characteristic of electricity prices, the model allows for a rich variety of volatility dynamics without restrictive positivity constraints, it can be estimated equation-by-equation by means of standard methods in widely available software, and Dynamic Conditional Correlations (DCCs) can - optionally - be estimated subsequent to the volatilities. In a study of the hourly day-ahead system prices at Nord Pool, we find extensive evidence of periodic volatility and volatility feedback, and that about half of the hours exhibit (positive) leverage. The strength of the ARCH, GARCH and leverage effects depend on the hour of the day. In only a single instance (at midnight) does the plain $\log$ - $\mathrm{GARCH}(1,1)$ perform better than the other specifications. We also find that the conditional correlations are time-varying, and that the conditional correlations are at their strongest in adjacent hours. This may have implications for risk-management, since it implies that portfolios risk is reduced if the degree of adjacency among the components of a portfolio prices is reduced.

\section{References}

Aielli, G. P. (2013). Dynamic Conditional Correlations: On Properties and Estimation. Journal of Business and Economic Statistics 31, 282-299. http: //dx.doi.org/10.1080/07350015.2013.771027.

Amado, C. and T. Terasvirta (2014a). Modelling Changes in the unconditional variance of long stock return series. Journal of Empirical Finance 25, 15-35.

Amado, C. and T. Terasvirta (2014b). Modelling volatility by variance decomposition. Journal of Econometrics 175, 142-153.

Bauwens, L., C. Hafner, and D. Pierret (2013). Multivariate Volatility Modelling of Electricity Futures. Journal of Applied Econometrics 28, 743-761.

Bunn, D. W. (2000). Forecasting Loads and Prices in Competitive Power Markets. Proceedings of the IEEE 88, 163-169.

Carnero, M. A., D. Pena, and E. Ruiz (2007). Effects of outliers on the identification and estimation of GARCH models. Journal of Time Series Analysis 28, 471-497. 
Creal, D., S. J. Koopmans, and A. Lucas (2011). A Dynamic Multivariate HeavyTailed Model for Time-Varying Volatilities and Correlations. Journal of Business and Economic Statistics 29, 552-563.

Duan, N. (1983). Smearing Estimate: A Nonparametric Retransformation Method. Journal of the Americal Statistical Association 78, pp. 605-610.

Dupuis, D. (2016). Electricity price dependence in new york state zones: A robust detrended correlation approach. Working Paper.

Engle, R. (2002). Dynamic Conditional Correlation: A Simple Class of Multivariate Generalized Autoregressive Conditional Heteroskedasticity Models. Journal of Business and Economic Statistics 20, 339-350.

Engle, R. F. and J. G. Rangel (2008). The Spline GARCH Model for Low Frequency Volatility and its Global Macroeconomic Causes. Review of Financial Studies 21, $1187-1222$.

Escribano, Á., J. I. Peña, and P. Villaplana (2002). Modelling Electricity Prices: International Evidence. UC3M Working Paper 02-27 in the Economic Series. Available as http://docubib.uc3m.es/WORKINGPAPERS/WE/we022708.pdf.

Escribano, A., J. I. Peña, and P. Villaplana (2011). Modelling Electricity Prices: International Evidence. Oxford Bulletin of Economics and Statistics 73, 622650. working paper version (2002): UC3M Working Paper 02-27 in the Economic Series.

Francq, C. and G. Sucarrat (2015). An Equation-by-Equation Estimator of a Multivariate Log-GARCH-X Model of Financial Returns. https://mpra.ub. uni-muenchen.de/67140/.

Francq, C. and J.-M. Zakoïan (2015). Estimating multivariate GARCH and stochastic correlation models equation by equation. Forthcoming in The Journal of the Royal Statistical Society. Series B. Working paper version: MPRA Paper No. 54250. Online at http://mpra.ub.uni-muenchen.de/54250/.

Gregory, A. and J. Reeves (2010). Estimation and inference in ARCH models in the presence of outliers. Journal of Financial Econometrics 8, 547-569.

Harvey, A. C. and G. Sucarrat (2014). EGARCH models with fat tails, skewness and leverage. Computational Statistics and Data Analysis 76, 320-338.

Hellström, J., J. Lundgren, and H. Yu (2012). Why do electricity prices jump? Empirical evidence from the nordic electricity market. Energy Economics 34, 1774-1781.

Janczura, J., S. Trück, R. Weron, and R. Wolff (2013). Identifying spikes and seasonal components in electricity spot price data: A guide to robust modeling. Energy Economics 38, 96-110. 
Knittel, C. R. and M. R. Roberts (2005). An empirical examination of restructured electricity prices. Energy Economics 27, 791-817.

Koopman, S. J., M. Ooms, and M. A. Carnero (2007). Periodic Seasonal REGARFIMA-GARCH Models for Daily Electricity Spot Prices. Journal of the American Statistical Association 102, 16-27.

Mazur, B. and M. Pipien (2012). On the Empirical Importance of Periodicity in the Volatility of Financial Returns - Time Varying GARCH as a Second Order APC(2) Process. Central European Journal of Economic Modelling and Econometrics 4, 95-116.

Nelson, D. B. (1991). Conditional Heteroskedasticity in Asset Returns: A New Approach. Econometrica 59, 347-370.

R Core Team (2014). R: A Language and Environment for Statistical Computing. Vienna, Austria: R Foundation for Statistical Computing.

Schwarz, G. (1978). Estimating the Dimension of a Model. The Annals of Statistics $6,461-464$.

Sucarrat, G. (2013). betategarch: Simulation, Estimation and Forecasting of Beta-Skew-t-EGARCH Models. The $R$ Journal 5, 137-147. http://journal. r-project.org/archive/2013-2/sucarrat.pdf.

Sucarrat, G. and Á. Escribano (2013). Unbiased QML Estimation of Log-GARCH Models in the Presence of Zero Returns. MPRA Paper No. 50699. Online at http://mpra.ub.uni-muenchen.de/50699/.

Sucarrat, G. and S. Grønneberg (2016). Models of Financial Return With TimeVarying Zero Probability. MPRA Paper No. 68931. Online at https://mpra.ub. uni-muenchen.de/68931/.

Sucarrat, G., S. Grønneberg, and Á. Escribano (2016). Estimation and Inference in Univariate and Multivariate Log-GARCH-X Models When the Conditional Density is Unknown. Computational Statistics and Data Analysis 100, 582-594. DOI: http://dx.doi.org/10.1016/j.csda.2015.12.005.

Van Bellegem, S. and R. Von Sachs (2004). Forecasting economic time-series with unconditional time-varying variance. International Journal of Forecasting 20, $611-627$.

Weron, R. (2014). Electricity price forecasting: A review of the state-of-the-art with a look into the future. International Journal of Forecasting 30, 1030-1081. 
Table 1: Estimation results of multivariate models (a)(e) for each equation $m=1, \ldots, 24$, see Section 3. Only selected parameter estimates reported

\begin{tabular}{|c|c|c|c|c|c|c|c|c|c|}
\hline$m$ & $R_{m}^{2}$ & Spec. & $\widehat{\alpha}_{m m .1}$ & $\widehat{\beta}_{m m .1}$ & $\widehat{\delta}_{m}$ & $\log L_{m}$ & $k_{m}$ & $B I C_{m}$ & $T$ \\
\hline \multirow[t]{5}{*}{1} & 0.05 & (a) & 0.145 & 0.785 & & 1696.179 & 3 & -2.1157 & 1593 \\
\hline & & (b) & & & & 1223.525 & 7 & -1.5037 & 1593 \\
\hline & & (c) & 0.150 & 0.779 & & 1761.886 & 9 & -2.1704 & 1593 \\
\hline & & (d) & 0.101 & 0.752 & & 1863.422 & 11 & -2.2900 & 1592 \\
\hline & & (e) & 0.100 & 0.759 & 0.462 & 1939.576 & 12 & $-2.3811^{*}$ & 1592 \\
\hline \multirow[t]{5}{*}{2} & 0.08 & (a) & 0.142 & 0.800 & & 1366.772 & 3 & -1.7021 & 1593 \\
\hline & & (b) & & & & 703.540 & 7 & -0.8509 & 1593 \\
\hline & & (c) & 0.151 & 0.787 & & 1443.756 & 9 & -1.7710 & 1593 \\
\hline & & (d) & 0.048 & 0.732 & & 1499.741 & 11 & -1.8332 & 1592 \\
\hline & & (e) & 0.039 & 0.806 & 0.517 & 1613.673 & 12 & $-1.9717^{*}$ & 1592 \\
\hline \multirow[t]{5}{*}{3} & 0.11 & (a) & 0.148 & 0.803 & & 972.495 & 3 & -1.2071 & 1593 \\
\hline & & (b) & & & & 377.325 & 7 & -0.4413 & 1593 \\
\hline & & (c) & 0.166 & 0.780 & & 1169.506 & 9 & -1.4266 & 1593 \\
\hline & & (d) & 0.095 & 0.748 & & 1214.471 & 11 & -1.4748 & 1592 \\
\hline & & (e) & 0.085 & 0.802 & 0.436 & 1304.535 & 12 & $-1.5833^{*}$ & 1592 \\
\hline \multirow[t]{5}{*}{4} & 0.13 & (a) & 0.145 & 0.808 & & 803.453 & 3 & -0.9948 & 1593 \\
\hline & & (b) & & & & 184.655 & 7 & -0.1994 & 1593 \\
\hline & & (c) & 0.168 & 0.778 & & 1016.636 & 9 & -1.2347 & 1593 \\
\hline & & (d) & 0.034 & 0.746 & & 1132.164 & 11 & -1.3714 & 1592 \\
\hline & & (e) & 0.040 & 0.812 & 0.374 & 1198.132 & 12 & $-1.4496^{*}$ & 1592 \\
\hline \multirow[t]{5}{*}{5} & 0.18 & (a) & 0.192 & 0.731 & & 591.917 & 3 & -0.7293 & 1593 \\
\hline & & (b) & & & & 214.012 & 7 & -0.2363 & 1593 \\
\hline & & (c) & 0.225 & 0.690 & & 1068.156 & 9 & -1.2994 & 1593 \\
\hline & & (d) & 0.161 & 0.646 & & 1092.515 & 11 & -1.3216 & 1592 \\
\hline & & (e) & 0.144 & 0.708 & 0.253 & 1136.385 & 12 & $-1.3720^{*}$ & 1592 \\
\hline \multirow[t]{5}{*}{6} & 0.34 & (a) & 0.408 & 0.023 & & 448.025 & 3 & -0.5486 & 1593 \\
\hline & & (b) & & & & 221.996 & 7 & -0.2463 & 1593 \\
\hline & & (c) & 0.210 & 0.695 & & 953.960 & 9 & -1.1560 & 1593 \\
\hline & & (d) & 0.125 & 0.612 & & 1105.194 & 11 & -1.3375 & 1592 \\
\hline & & (e) & 0.116 & 0.652 & 0.141 & 1137.632 & 12 & $-1.3736^{*}$ & 1592 \\
\hline \multirow[t]{5}{*}{7} & 0.46 & (a) & 0.342 & 0.026 & & 536.814 & 3 & -0.6601 & 1593 \\
\hline & & (b) & & & & 569.559 & 7 & -0.6827 & 1593 \\
\hline & & (c) & 0.206 & 0.654 & & 1078.526 & 9 & -1.3124 & 1593 \\
\hline & & (d) & 0.125 & 0.621 & & 1118.880 & 11 & $-1.3547^{*}$ & 1592 \\
\hline & & (e) & 0.127 & 0.615 & -0.025 & 1113.663 & 12 & -1.3435 & 1592 \\
\hline \multirow[t]{4}{*}{8} & 0.49 & (a) & 0.230 & 0.247 & & 473.888 & 3 & -0.5811 & 1593 \\
\hline & & (b) & & & & 582.832 & 7 & -0.6993 & 1593 \\
\hline & & (c) & 0.146 & 0.705 & & 800.839 & 9 & -0.9638 & 1593 \\
\hline & & (d) & 0.071 & 0.644 & & 857.150 & 11 & $-1.0259^{*}$ & 1592 \\
\hline
\end{tabular}

Table continues on next page. Explanatory note at the end of table. 


\begin{tabular}{|c|c|c|c|c|c|c|c|c|c|}
\hline$m$ & $R_{m}^{2}$ & Spec. & $\widehat{\alpha}_{m m .1}$ & $\widehat{\beta}_{m m .1}$ & $\widehat{\delta_{m}}$ & $\log L_{m}$ & $k_{m}$ & $B I C_{m}$ & $T$ \\
\hline \multirow{3}{*}{9} & \multirow{3}{*}{0.50} & (e) & 0.069 & 0.630 & -0.190 & 824.509 & 12 & -0.9802 & 1592 \\
\hline & & (a) & 0.112 & 0.795 & & 702.853 & 3 & -0.8685 & 1593 \\
\hline & & (b) & & & & 636.460 & 7 & -0.7667 & 1593 \\
\hline \multirow{7}{*}{10} & \multirow{7}{*}{0.48} & (c) & 0.120 & 0.784 & & 841.824 & 9 & -1.0152 & 1593 \\
\hline & & (d) & 0.080 & 0.753 & & 877.438 & 11 & $-1.0514^{*}$ & 1592 \\
\hline & & (e) & 0.083 & 0.745 & -0.072 & 862.803 & 12 & -1.0284 & 1592 \\
\hline & & (a) & 0.106 & 0.820 & & 1169.681 & 3 & -1.4546 & 1593 \\
\hline & & (b) & & & & 958.053 & 7 & -1.1704 & 1593 \\
\hline & & (c) & 0.111 & 0.813 & & 1218.310 & 9 & -1.4879 & 1593 \\
\hline & & (d) & 0.061 & 0.801 & & 1261.253 & 11 & $-1.5335^{*}$ & 1592 \\
\hline \multirow{6}{*}{11} & \multirow{3}{*}{0.44} & (e) & 0.061 & 0.800 & -0.050 & 1254.686 & 12 & -1.5207 & 1592 \\
\hline & & (a) & 0.087 & 0.848 & & 1436.812 & 3 & -1.7900 & 1593 \\
\hline & & (b) & & & & 1230.942 & 7 & -1.5130 & 1593 \\
\hline & & (c) & 0.092 & 0.840 & & 1478.363 & 9 & -1.8144 & 1593 \\
\hline & & (d) & -0.061 & 0.815 & & 1588.854 & 11 & $-1.9451^{*}$ & 1592 \\
\hline & & (e) & -0.061 & 0.815 & -0.026 & 1586.327 & 12 & -1.9373 & 1592 \\
\hline \multirow[t]{5}{*}{12} & 0.40 & (a) & 0.096 & 0.837 & & 1652.884 & 3 & -2.0613 & 1593 \\
\hline & & (b) & & & & 1348.482 & 7 & -1.6606 & 1593 \\
\hline & & (c) & 0.103 & 0.827 & & 1675.852 & 9 & -2.0624 & 1593 \\
\hline & & (d) & 0.090 & 0.808 & & 1711.472 & 11 & $-2.0991^{*}$ & 1592 \\
\hline & & (e) & 0.090 & 0.808 & -0.030 & 1711.643 & 12 & -2.0947 & 1592 \\
\hline \multirow[t]{5}{*}{13} & 0.39 & (a) & 0.108 & 0.817 & & 1750.552 & 3 & -2.1839 & 1593 \\
\hline & & (b) & & & & 1463.161 & 7 & -1.8046 & 1593 \\
\hline & & (c) & 0.115 & 0.805 & & 1786.314 & 9 & -2.2010 & 1593 \\
\hline & & (d) & 0.026 & 0.759 & & 1845.398 & 11 & -2.2674 & 1592 \\
\hline & & (e) & 0.025 & 0.762 & 0.066 & 1850.175 & 12 & $-2.2688^{*}$ & 1592 \\
\hline \multirow[t]{5}{*}{14} & 0.42 & (a) & 0.116 & 0.801 & & 1736.312 & 3 & -2.1660 & 1593 \\
\hline & & (b) & & & & 1497.177 & 7 & -1.8473 & 1593 \\
\hline & & (c) & 0.127 & 0.786 & & 1794.275 & 9 & -2.2110 & 1593 \\
\hline & & (d) & 0.068 & 0.757 & & 1836.675 & 11 & -2.2564 & 1592 \\
\hline & & (e) & 0.069 & 0.767 & 0.103 & 1850.518 & 12 & $-2.2692^{*}$ & 1592 \\
\hline \multirow[t]{5}{*}{15} & 0.41 & (a) & 0.104 & 0.800 & & 1647.765 & 3 & -2.0549 & 1593 \\
\hline & & (b) & & & & 1448.164 & 7 & -1.7858 & 1593 \\
\hline & & (c) & 0.114 & 0.787 & & 1698.284 & 9 & -2.0905 & 1593 \\
\hline & & (d) & 0.017 & 0.805 & & 1769.745 & 11 & -2.1724 & 1592 \\
\hline & & (e) & 0.018 & 0.804 & 0.042 & 1777.901 & 12 & $-2.1780^{*}$ & 1592 \\
\hline \multirow[t]{5}{*}{16} & 0.40 & (a) & 0.103 & 0.819 & & 1655.581 & 3 & -2.0647 & 1593 \\
\hline & & (b) & & & & 1428.192 & 7 & -1.7607 & 1593 \\
\hline & & (c) & 0.113 & 0.804 & & 1734.833 & 9 & -2.1364 & 1593 \\
\hline & & (d) & 0.045 & 0.748 & & 1760.855 & 11 & $-2.1612^{*}$ & 1592 \\
\hline & & (e) & 0.045 & 0.744 & -0.019 & 1757.760 & 12 & -2.1527 & 1592 \\
\hline \multirow[t]{2}{*}{17} & 0.35 & (a) & 0.119 & 0.792 & & 1574.735 & 3 & -1.9632 & 1593 \\
\hline & & (b) & & & & 1326.363 & 7 & -1.6328 & 1593 \\
\hline
\end{tabular}

Table continues on next page. Explanatory note at the end of table. 


\begin{tabular}{|c|c|c|c|c|c|c|c|c|c|}
\hline$m$ & $R_{m}^{2}$ & Spec. & $\widehat{\alpha}_{m m .1}$ & $\widehat{\beta}_{m m .1}$ & $\widehat{\delta}_{m}$ & $\log L_{m}$ & $k_{m}$ & $B I C_{m}$ & $T$ \\
\hline & & (c) & 0.127 & 0.782 & & 1583.191 & 9 & -1.9460 & 1593 \\
\hline & & (d) & 0.042 & 0.746 & & 1675.591 & 11 & $-2.0541^{*}$ & 1592 \\
\hline & & (e) & 0.041 & 0.749 & 0.025 & 1678.602 & 12 & -2.0532 & 1592 \\
\hline \multirow[t]{5}{*}{18} & 0.30 & (a) & 0.118 & 0.833 & & 1649.697 & 3 & -2.0573 & 1593 \\
\hline & & (b) & & & & 1198.336 & 7 & -1.4721 & 1593 \\
\hline & & (c) & 0.123 & 0.827 & & 1663.243 & 9 & -2.0465 & 1593 \\
\hline & & (d) & 0.069 & 0.815 & & 1719.584 & 11 & $-2.1093^{*}$ & 1592 \\
\hline & & (e) & 0.068 & 0.814 & -0.070 & 1712.633 & 12 & -2.0960 & 1592 \\
\hline \multirow[t]{5}{*}{19} & 0.23 & (a) & 0.114 & 0.830 & & 1784.389 & 3 & -2.2264 & 1593 \\
\hline & & (b) & & & & 1320.064 & 7 & -1.6249 & 1593 \\
\hline & & (c) & 0.118 & 0.825 & & 1783.915 & 9 & -2.1980 & 1593 \\
\hline & & (d) & 0.026 & 0.791 & & 1868.741 & 11 & $-2.2967^{*}$ & 1592 \\
\hline & & (e) & 0.026 & 0.791 & -0.025 & 1868.236 & 12 & -2.2915 & 1592 \\
\hline \multirow[t]{5}{*}{20} & 0.20 & (a) & 0.130 & 0.778 & & 2051.643 & 3 & -2.5619 & 1593 \\
\hline & & (b) & & & & 1558.624 & 7 & -1.9244 & 1593 \\
\hline & & (c) & 0.135 & 0.770 & & 2057.749 & 9 & -2.5418 & 1593 \\
\hline & & (d) & 0.071 & 0.721 & & 2099.335 & 11 & $-2.5864^{*}$ & 1592 \\
\hline & & (e) & 0.071 & 0.721 & -0.013 & 2098.817 & 12 & -2.5811 & 1592 \\
\hline \multirow[t]{5}{*}{21} & 0.16 & (a) & 0.109 & 0.807 & & 2260.769 & 3 & -2.8245 & 1593 \\
\hline & & (b) & & & & 1815.880 & 7 & -2.2474 & 1593 \\
\hline & & (c) & 0.113 & 0.801 & & 2269.980 & 9 & -2.8083 & 1593 \\
\hline & & (d) & 0.018 & 0.755 & & 2341.591 & 11 & -2.8908 & 1592 \\
\hline & & (e) & 0.017 & 0.758 & 0.059 & 2347.321 & 12 & $-2.8933^{*}$ & 1592 \\
\hline \multirow[t]{5}{*}{22} & 0.13 & (a) & 0.132 & 0.781 & & 2441.839 & 3 & -3.0518 & 1593 \\
\hline & & (b) & & & & 2022.447 & 7 & -2.5068 & 1593 \\
\hline & & (c) & 0.136 & 0.775 & & 2446.624 & 9 & -3.0301 & 1593 \\
\hline & & (d) & 0.080 & 0.750 & & 2489.917 & 11 & -3.0771 & 1592 \\
\hline & & (e) & 0.079 & 0.754 & 0.090 & 2499.693 & 12 & $-3.0847^{*}$ & 1592 \\
\hline \multirow[t]{5}{*}{23} & 0.10 & (a) & 0.137 & 0.761 & & 2448.579 & 3 & -3.0603 & 1593 \\
\hline & & (b) & & & & 2014.287 & 7 & -2.4965 & 1593 \\
\hline & & (c) & 0.141 & 0.755 & & 2478.169 & 9 & -3.0697 & 1593 \\
\hline & & (d) & 0.078 & 0.740 & & 2541.112 & 11 & -3.1414 & 1592 \\
\hline & & (e) & 0.079 & 0.742 & 0.044 & 2545.099 & 12 & $-3.1418^{*}$ & 1592 \\
\hline
\end{tabular}

Table continues on next page. Explanatory note at the end of table. 


\begin{tabular}{cccccccccc}
\hline$m$ & $R_{m}^{2}$ & Spec. & $\widehat{\alpha}_{m m .1}$ & $\widehat{\beta}_{m m .1}$ & $\widehat{\delta}_{m}$ & $\log L_{m}$ & $k_{m}$ & $B I C_{m}$ & $T$ \\
\hline 24 & 0.10 & (a) & 0.131 & 0.818 & & 2048.593 & 3 & $-2.5581^{*}$ & 1593 \\
& & (b) & & & & 1356.737 & 7 & -1.6710 & 1593 \\
& & (c) & 0.133 & 0.815 & & 2001.696 & 9 & -2.4715 & 1593 \\
& & (d) & 0.119 & 0.796 & & 1967.751 & 11 & -2.4211 & 1592 \\
& & (e) & 0.113 & 0.808 & 0.250 & 2038.426 & 12 & -2.5053 & 1592 \\
\hline
\end{tabular}

$R_{m}^{2}$, the $R$-squared of conditional mean equation $m$. Spec., the log-GARCH specification in question, see Section 3. $\widehat{\alpha}_{m m .1}, \widehat{\beta}_{m m .1}$ and $\widehat{\delta}_{m}$, the ARCH, GARCH and asymmetry/leverage estimates, respectively. $\log L_{m}$, the Gaussian log-likelihood of equation $m$ in question. $k_{m}$, the number of parameters in the log-GARCH specification in question. $B I C_{m}$, the value of the Schwarz (1978) information criterion for the equation $m$ in question in terms of the average log-likelihood. $T$, the number of observations. All computations in $R$ (R Core Team (2014)). 

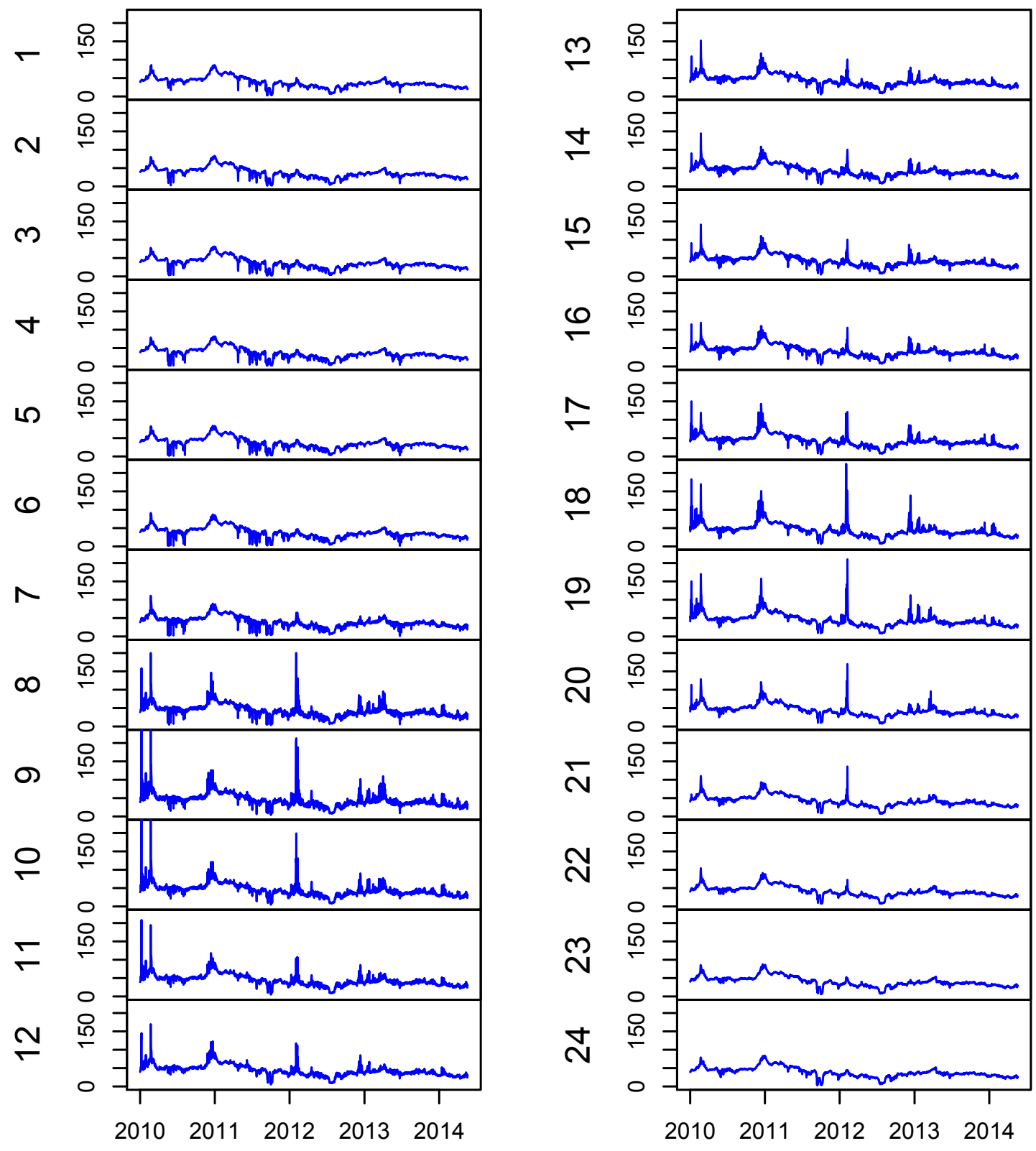

Figure 1: Hourly $(m=1, \ldots, 24)$ day-ahead system prices in Euros per $\mathrm{kw} / \mathrm{h}$ at Nord Pool from 1 January 2010 to 20 May 2014 ( $T=1601$ observations before differencing and lagging), see Section 3.1. 

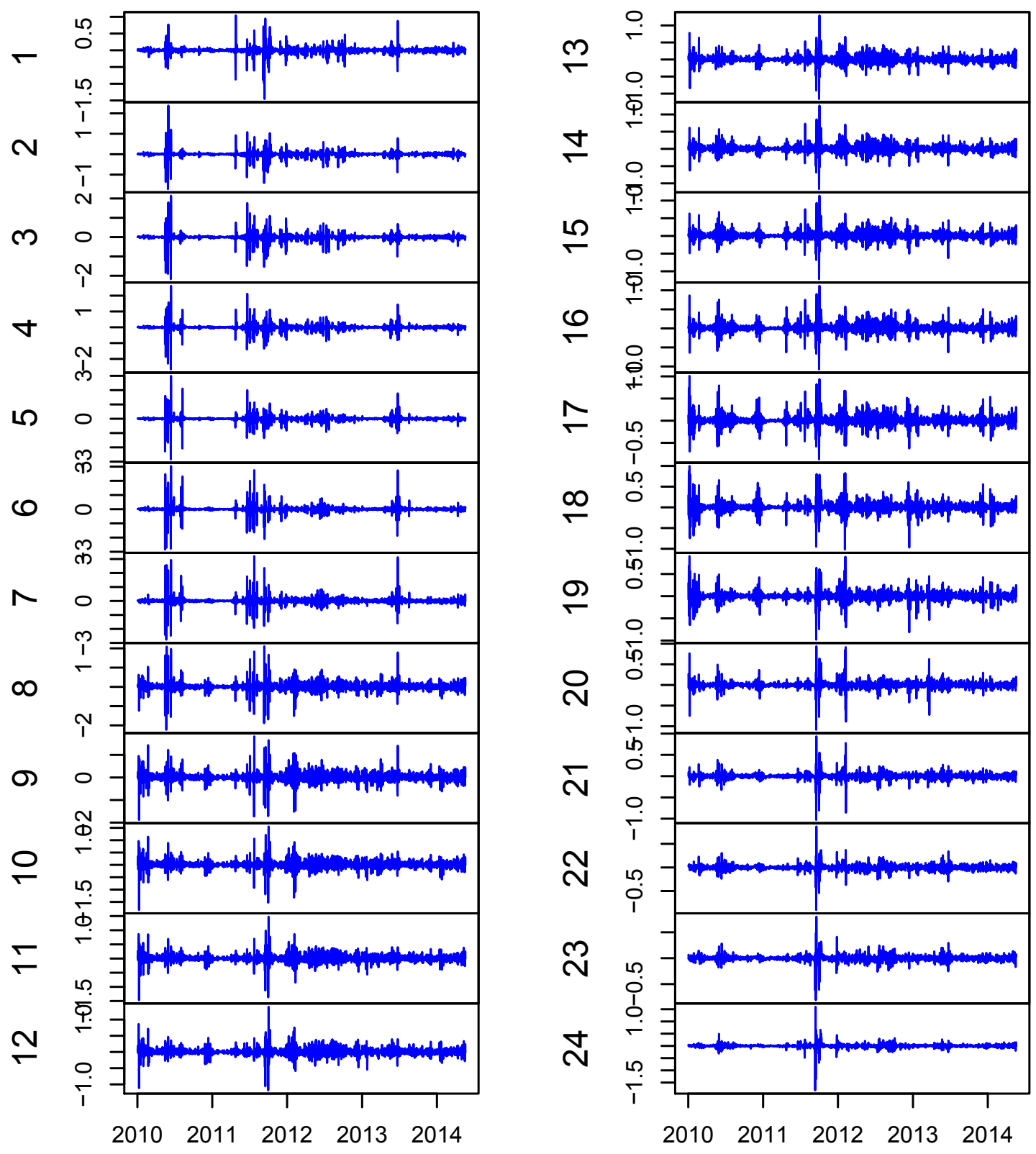

Figure 2: Hourly $(m=1, \ldots, 24)$ day-ahead log-returns of system prices at Nord Pool from 1 January 2010 to 20 May 2014, see Section 3.1. 


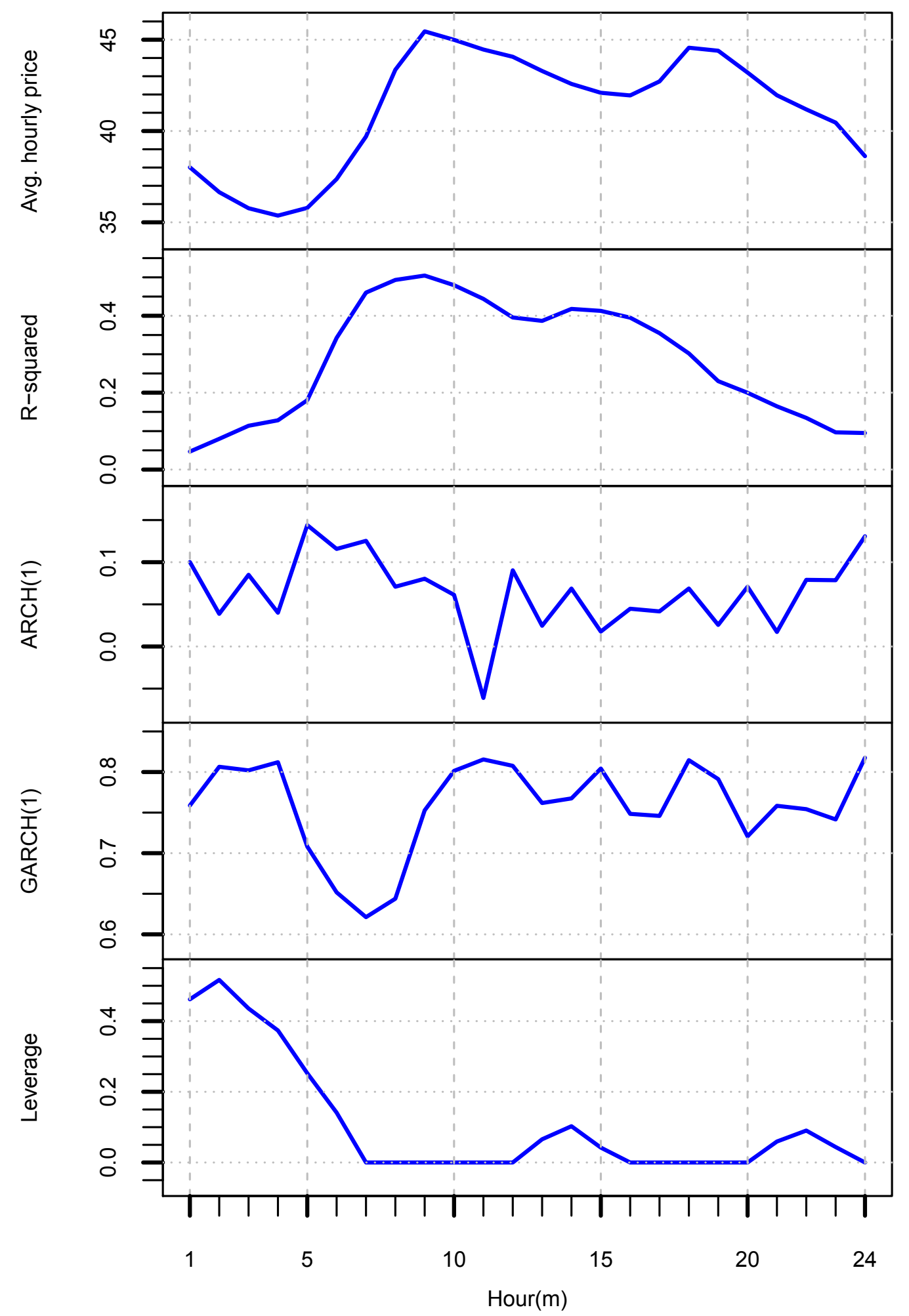

Figure 3: The average hourly price in Euros (top graph), the R-squared of the conditional mean equations (second graph), and the estimated $\mathrm{ARCH}(1), \mathrm{GARCH}(1)$ and leverage parameters of the best specification (according to BIC) in each $m$ (third, fourth and bottom graphs). The best specification in hour $m$ is identified by means of an asterisk * to the right of its BIC value in Table 1. 

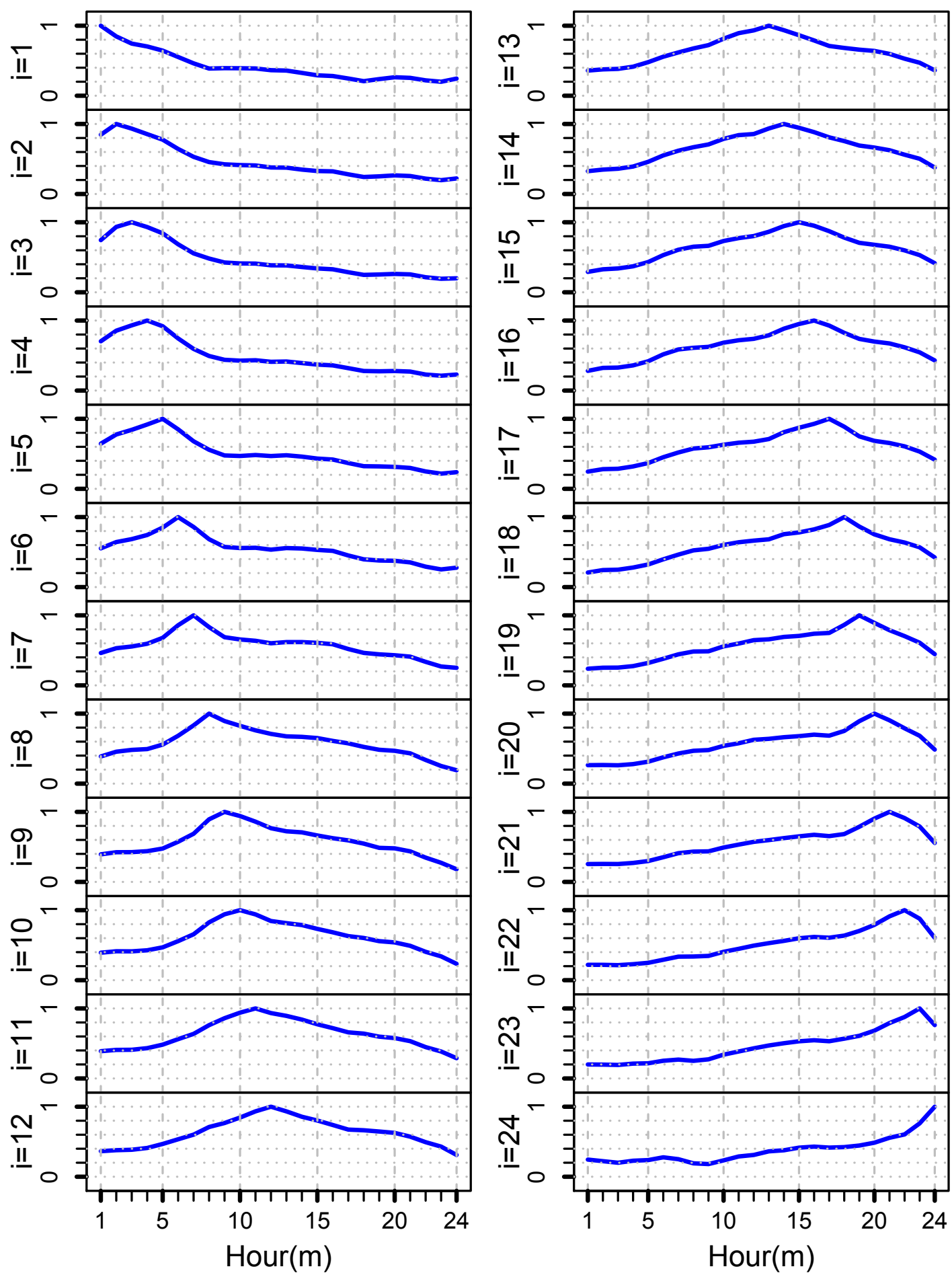

Figure 4: Estimates of the unconditional correlations $E\left(\eta_{i t} \eta_{m t}\right), i=1, \ldots, 24$ and $m=1, \ldots, 24$, see Section 3.3. 

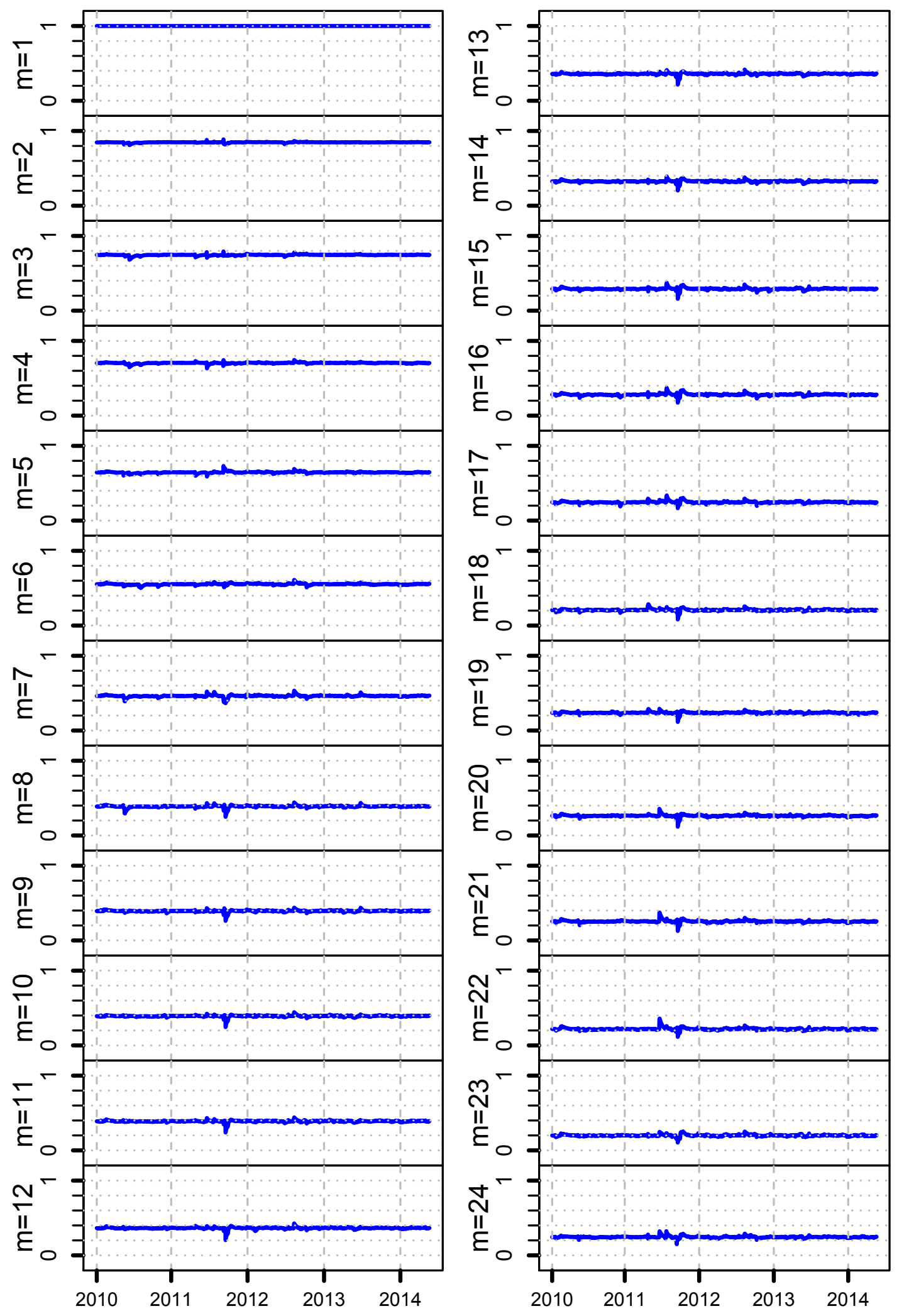

Figure 5: Estimates of a subset (the first 24) of the conditional correlations; $\widehat{E}_{t-1}\left(\eta_{1 t} \eta_{m t}\right)$ for $m=1, \ldots, 24$, see Section 3.3. 